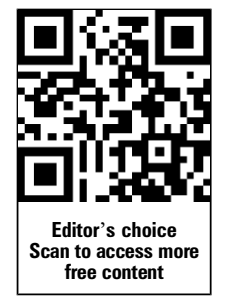

${ }^{1}$ Department of Mechanical Engineering, Melbourne School of Engineering, The University of Melbourne, Melbourne, Victoria, Australia

${ }^{2}$ Division of Physiotherapy, School of Health and Rehabilitation Sciences, The University of Queensland, Brisbane, Queensland Australia

${ }^{3}$ School of Physiotherapy and Exercise Science, Griffith University, Gold Coast, Queensland, Australia ${ }^{4}$ Department of General Practice, Erasmus MC Medical University, Rotterdam, The Netherlands

${ }^{5}$ Department of Orthopaedics, Erasmus MC Medical University, Rotterdam, The Netherlands

\section{Correspondence to}

Dr Natalie Collins, Department of Mechanical Engineering, Melbourne School of Engineering, The University of Melbourne, VIC 3010,

Australia;

n.collins@unimelb.edu.au

Received 17 August 2012 Revised 10 October 2012 Accepted 10 November 2012 Published Online First 13 December 2012

\begin{tabular}{l}
\hline To cite: Collins NJ, Bierma- \\
Zeinstra SMA, Crossley KM, \\
et al. Br J Sports Med \\
2013;47:227-233. \\
\hline
\end{tabular}

\title{
Prognostic factors for patellofemoral pain: a multicentre observational analysis
}

\author{
Natalie J Collins, ${ }^{1,2,3}$ Sita M A Bierma-Zeinstra, ${ }^{4,5}$ Kay M Crossley, ${ }^{1,2}$ \\ Robbart L van Linschoten, ${ }^{4}$ Bill Vicenzino, ${ }^{2}$ Marienke van Middelkoop ${ }^{4}$
}

\section{ABSTRACT \\ Objectives Describe proportions of individuals with patellofemoral pain (PFP) with an unfavourable recovery over 12 months; identify clinical predictors of poor recovery at 3 and 12 months; and determine baseline values of predictors that identify those with poor 12-month prognosis.}

Methods An observational analysis utilised data from 310 individuals with PFP enrolled in two randomised clinical trials. Thirteen baseline variables (participant, PFP, study characteristics) were investigated for their prognostic ability. Pain, function and global recovery were measured at 3 and 12 months. Multivariate backward stepwise regression analyses (treatmentadjusted, $p<0.10$ ) were performed for each follow-up measure. Receiver operator characteristic curves identified cut-points associated with unfavourable recovery at 12 months.

Results $55 \%$ and $40 \%$ of participants had an unfavourable recovery at 3 and 12 months, respectively. Longer baseline pain duration was significantly associated with poor 3-month and 12-month recovery on measures of pain severity ( $\beta 11.36$ to 24.94), Anterior Knee Pain (AKP) Scale (-4.44 to -11.33) and global recovery (OR: 2.32 to 6.11). Greater baseline pain severity and lower AKP Scale score were significantly associated with poor recovery on multiple measures $(p<0.05)$. Baseline duration $>2$ months and AKP Scale score $<70 / 100$ were associated with unfavourable 12-month recovery.

Conclusions A substantial number of individuals with PFP have an unfavourable recovery over 12 months, irrespective of intervention. Knee pain duration $>2$ months is the most consistent prognostic indicator, followed by AKP Scale score $<70$. Sports medicine practitioners should utilise interventions with known efficacy in reducing PFP, and promote early intervention to maximise prognosis.

Trial registration Australian study: Australian Clinical Trials Registry (ACTRN012605000463673), ClinicalTrials. gov (NCT00118521); Dutch study: International Standard Randomised Controlled Trial Number Register (ISRCTN83938749)

\section{BACKGROUND}

Patellofemoral pain (PFP) is a common condition that imposes a substantial burden on individuals and healthcare systems internationally. Those affected experience an insidious onset anterior or retropatellar knee pain, which is aggravated by activities that load the patellofemoral joint (PFJ) (eg, squatting, stair ambulation and running). ${ }^{1}{ }^{2}$ This can affect participation in daily work and exercise, with important implications for the prevention of conditions such as cardiovascular disease, diabetes and osteoporosis. Prospective studies show that PFP is not self-limiting, with persistent symptoms up to 20 years, ${ }^{3}$ while preliminary evidence suggests that PFP may be a precursor to PFJ osteoarthritis. ${ }^{4}$ Furthermore, individual responses to efficacious interventions are variable, with a proportion of people having unfavourable outcomes. ${ }^{25}$

In healthcare, prognosis often refers to the likelihood of an individual experiencing a poor outcome over time, based on demographic, diagnostic or comorbid characteristics. ${ }^{6-8}$ Identification of clinically meaningful prognostic factors for PFP would provide practitioners with information regarding characteristics that may predict an individual's prognosis, irrespective of subsequent treatment. This can also be used to inform patients regarding their likely clinical course and facilitate more realistic expectations of treatment outcomes. ${ }^{9}$ To gain the most accurate representation of prognosis and clinical applicability, a multivariate approach is necessary, which provides probabilities regarding outcomes for different combinations of predictor variables. ${ }^{7}$ Four prospective studies have utilised multivariate regression modelling for PFP, identifying older age, greater height, longer symptom duration, lower Anterior Knee Pain (AKP) Scale score (indicating worse symptoms), greater pain during the patellar apprehension test, and longer vastus medialis obliquus reflex response time as prognostic indicators. ${ }^{10-13}$ However, only one of these studies controlled for treatment received during the observation period, ${ }^{10}$ which is a vital component of multivariate analysis given its potential influence on prognosis.

The current study utilised two international PFP cohorts to address three objectives: (1) describe the proportion of individuals with PFP who experience an unfavourable recovery over 12 months; (2) identify clinically applicable factors that predict poor recovery at 3 and 12 months and (3) determine baseline values of predictor variables to assist clinicians in identifying those who may have a poor 12-month prognosis.

\section{METHODOLOGY}

\section{Data source}

Prospective data were derived from two randomised clinical trials (RCTs) that investigated the effectiveness of conservative interventions for PFP. $^{2} 5$ Study protocols have been detailed previously. ${ }^{14} 15$ The pooled dataset included 310 participants with PFP (Australian RCT $n=179$; Dutch RCT $\mathrm{n}=131$ ), with follow-up over 12 months. Both RCTs had institutional ethics approval 
(The University of Queensland's Medical Research Ethics Committee; ${ }^{2}$ The Erasmus Medical University, Rotterdam ${ }^{5}$ ).

\section{Participants}

The pooled sample comprised individuals recruited via primary practitioner referral (general practitioner, sports physician), and by self-referral from community advertising. ${ }^{2}$ In both studies, volunteers were included if they had an insidious onset of anterior knee or retropatellar pain greater than 6 weeks duration, aggravated by at least two activities that load the PFJ (eg prolonged sitting, kneeling, squatting, running, cycling and stair ambulation). Exclusion criteria common to both studies were the presence of other defined knee pathology (eg osteoarthritis, patellar tendinopathy and Osgood-Schlatters disease), and physiotherapy intervention within the preceding year. The Australian RCT also excluded individuals who rated their worst PFP over the previous week less than $30 \mathrm{~mm}$ on a $100 \mathrm{~mm}$ visual analogue scale (VAS); concomitant injury or pain from the hip or lumbar spine; foot conditions precluding the use of foot orthoses; use of foot orthoses within the previous year; current anti-inflammatory drug use; and allergy to adhesive sports tape. Furthermore, while the Dutch RCT recruited participants as young as 14 years, the Australian RCT had a minimum age of 18 years.

\section{Interventions}

Both studies randomly allocated participants to interventions. Participants in the Australian RCT received one of four intervention protocols: prefabricated foot orthoses $(n=46)$; flat shoe inserts $(n=44)$; multimodal physiotherapy consisting of patellar mobilisation, patellar taping, vasti retraining and hip and hamstring stretches $(n=45)$; and foot orthoses plus physiotherapy $(n=44){ }^{15}$ Physiotherapists administered all interventions, once a week for 6 weeks. The Dutch trial compared two intervention protocols, exercise therapy $(n=65)$ and usual care $(n=66) .{ }^{14}$ Both groups received standardised information regarding PFP and advice to avoid aggravating activities, from their referring practitioner. In addition, those assigned to exercise therapy completed a programme of strength, flexibility and balance exercises, supervised by a physiotherapist during nine sessions over 6 weeks, and were advised to practise at home for an additional 6 weeks.

\section{Outcome measures and potential predictive variables}

Patient-reported measures of pain severity, function and global recovery were assessed over 12 months. Pain severity was measured as worst pain or pain during activity, on a $100 \mathrm{~mm}$ VAS or 11-point numerical rating scale $(0-10)$, respectively. Both trials administered the AKP Scale $(0-100)^{16}$ and Functional Index Questionnaire (FIQ) (0-16). ${ }^{17}$ Global recovery was measured on a five-point Likert scale in the Australian RCT ('marked improvement' to 'marked worsening'), while the Dutch study used a seven-point Likert scale ('completely recovered' to 'worse than ever'). To allow data pooling, these were dichotomised into 'favourable recovery' (defined as 'completely recovered', 'strongly recovered' or 'marked improvement') and 'unfavourable recovery' ('moderate improvement' to 'worse than ever'). ${ }^{18}{ }^{19}$ Pain severity, AKP Scale and FIQ were measured at baseline, and all measures conducted at 6 weeks, 3 and 12 months. The Dutch trial also included 6-month and 9-month follow-ups. Since differences in effect can be time dependent, both short-term and long-term prognostic factors were investigated. Short-term was defined as 3-month follow-up, with long-term follow-up defined as 12 months.
Potential prognostic factors were selected from baseline data, primarily from self-reported questionnaires. To comply with recommendations of at least 10 events per variable investigated, ${ }^{20}$ the number of candidate variables was restricted to 13. Participant characteristics evaluated for their predictive ability were age, gender, body mass index (BMI), work type (sedentary/active) and sport participation (yes/no). PFP variables were knee pain duration (1-2, 2-6, 6-12 and $\geq 12$ months), bilaterality and baseline scores of pain and function (worst pain or pain during activity; usual pain or pain at rest; AKP Scale; FIQ). Study characteristics were recruitment method (health professional; self-referral), and allocation to preferred treatment (yes/ no/no preference). Possible values and categories of the candidate predictor variables are presented in table 1 . To ensure optimal statistical strength, we refrained from categorising continuous variables. ${ }^{21}$

\section{Data management and statistical analyses}

All statistical analyses were performed using SPSS V.17.0 (SPSS Inc, Chicago, Illinois, USA). Baseline data for each candidate variable were presented as means and standard deviations for continuous data, and numbers and percentages for categorical data. Independent $t$ tests and Pearson $\chi^{2}$ tests were used to evaluate between-study differences in continuous and categorical baseline variables, respectively. Descriptive statistics were used to describe the condition at short-term and long-term follow-up.

Candidate variables were entered into multivariate backward stepwise regression analyses, for each measure at 3 and 12 months. $^{22}$ Linear regression was used for outcomes of pain, AKP Scale and FIQ, and logistic regression for the dichotomous measure of global recovery. Variables with the highest $\mathrm{p}$ values were removed one at a time (Wald test), until all remaining variables were significant at $\mathrm{p}<0.10$. Treatment group was included as a covariate in all multivariate models initially, but was removed in the backwards stepwise process if not significant. Associations within each multivariate model were regarded as significant at $\mathrm{p} \leq 0.05$. The strength of the predictive ability of identified factors in each multivariate model was determined using OR for dichotomous measures (global recovery) and unstandardised regression coefficients $(\beta)$ for continuous measures (pain, AKP Scale, FIQ), with 95\% CI. Overall performance of final models was evaluated with Nagelskerke's $\mathrm{R}^{2}$, which estimates explained variation of the model. ${ }^{23}$ Discrimination for the dichotomous measure of success was assessed by calculating the area under the receiver operator characteristic (ROC) curve, to evaluate how well each model distinguished patients who perceived themselves as recovered from those who did not. ${ }^{24} 25$

For categorical variables, categories that were predictive of unfavourable recovery at 12 months were obtained from multivariate analyses. Cut-off values for continuous baseline variables that were predictive of unfavourable 12-month recovery were determined by plotting ROC curves using sensitivity and specificity values for all possible cut-points. The point on the ROC curve nearest the upper left-hand corner was selected as the cut-off value for unfavourable recovery.

\section{RESULTS}

Baseline characteristics are presented in table 1. Compared to the Australian RCT, participants in the Dutch trial were significantly younger (mean difference 5.3 years, 95\% CI 3.6 to 6.9) and had a lower BMI (1.73, 0.74 to 2.73$)$, greater participation in sport $(p=0.022)$ and greater unemployment $(p<0.001)$. On 
Table 1 Baseline characteristics of study participants

\begin{tabular}{|c|c|c|c|}
\hline Variable & RCT Australia (n=179) & RCT the Netherlands $(n=131)$ & Overall $(n=310)$ \\
\hline Age (years) & $29.3(5.8)$ & $24.0(8.2)$ & $27.1(7.4)^{*}$ \\
\hline Gender (female) (\%) & $100(55.9)$ & $84(64.1)$ & $184(59.4)$ \\
\hline BMI $\left(\mathrm{kg} / \mathrm{m}^{2}\right)$ & $24.8(5.1)$ & $23.0(3.7)$ & $24.0(4.6)^{*}$ \\
\hline \multicolumn{4}{|l|}{ Work status (\%) } \\
\hline Not employed & $28(15.6)$ & $52(40.9)$ & $80(25.8)^{*}$ \\
\hline Sedentary & $100(55.9)$ & $28(21.4)$ & $128(41.3)$ \\
\hline Active & $50(27.9)$ & $47(35.9)$ & $97(31.3)$ \\
\hline Unknown & $1(0.6)$ & $4(3.1)$ & $5(1.6)$ \\
\hline Sport participant (\%) & $118(65.9)$ & $102(77.9)$ & $220(71.0)^{*}$ \\
\hline \multicolumn{4}{|l|}{ Duration of knee pain (\%) } \\
\hline $1-2$ months & $8(4.5)$ & $50(38.2)$ & $58(18.7)^{\star}$ \\
\hline 2-6 months & $17(9.5)$ & $39(29.8)$ & $56(18.1)$ \\
\hline $6-12$ months & $30(16.8)$ & $19(14.5)$ & $49(15.8)$ \\
\hline$>12$ months & $120(67.0)$ & $23(17.6)$ & $143(46.1)$ \\
\hline Unknown & $4(2.2)$ & & \\
\hline Bilateral pain (\%) & $102(57.0)$ & $79(60.3)$ & $181(58.4)$ \\
\hline \multicolumn{4}{|l|}{ Allocated preferred treatment } \\
\hline Not allocated & $66(36.9 \%)$ & $52(39.7 \%)$ & $118(38.1 \%)$ \\
\hline Allocated & $55(30.7 \%)$ & $50(38.2 \%)$ & $105(33.9 \%)$ \\
\hline No treatment preference & $38(21.2 \%)$ & $28(21.4 \%)$ & $66(21.3 \%)$ \\
\hline Unknown & $20(11.2 \%)$ & $1(0.8 \%)$ & $21(6.8 \%)$ \\
\hline Recruitment (health professional), \% & $2(1.1 \%)$ & $131(100 \%)$ & $133(43.3 \%)^{*}$ \\
\hline Usual or resting pain (VAS/100) & $36.3(16.6)$ & $40.8(22.8)$ & $38.2(19.5)$ \\
\hline Worst or activity-related pain (VAS/100) & $60.5(15.9)$ & $61.5(22.1)$ & $60.9(18.7)$ \\
\hline Symptoms and function (AKP Scale/100) & $71.5(9.8)$ & $65.1(14.5)$ & $68.8(12.4)^{*}$ \\
\hline Function (FIQ/16) & $9.8(2.1)$ & $9.5(2.5)$ & $9.7(2.3)$ \\
\hline \multicolumn{4}{|l|}{ Treatment } \\
\hline Usual care & - & $66(50.4 \%)$ & $66(21.3 \%)$ \\
\hline Exercise therapy & - & $65(49.6 \%)$ & $65(21.0 \%)$ \\
\hline Physiotherapy & $45(25.1 \%)$ & - & $45(14.5 \%)$ \\
\hline Foot orthoses & $46(25.7 \%)$ & - & $46(14.8 \%)$ \\
\hline Flat inserts & $44(24.6 \%)$ & - & $44(14.2 \%)$ \\
\hline Physiotherapy+foot orthoses & $44(24.6 \%)$ & - & $44(14.2 \%)$ \\
\hline
\end{tabular}

average, PFP duration was shorter for Dutch participants $(\mathrm{p}<0.001)$, and they reported lower AKP Scale scores $(6.4,1.5$ to 3.5$)$, indicating worse symptoms. The recruitment source for each trial also reflected differences in study methodologies, with the Dutch RCT recruiting significantly more participants from health professionals $(\mathrm{p}<0.001)$.

\section{Outcomes}

Fifty-five per cent of participants reported unfavourable outcome at 3 months on the dichotomised measure of global recovery (170/310) (table 2). This decreased to 40\% (126/310) at 12 months. Mean pain severity (worst or activity-related pain) decreased from $35 / 100$ at 3 months to $26 / 100$ at 12 months. Small improvements were seen on the AKP Scale and FIQ between 3 and 12 months.

\section{Prognostic indicators}

Pain severity

The multivariate model for worst or activity-related pain severity at 3 months revealed that longer PFP duration (612 months: $\beta 12.33$, 95\% CI 3.56 to 21.09 ; > 12 months: $11.36,3.96$ to 18.77 ), greater baseline severity of worst or activity-related pain $(0.45,0.28$ to 0.62$)$ and lower baseline
AKP Scale score $(-0.33,-0.59$ to -0.06$)$ were significantly associated with greater pain severity (table 3 ). The model explained $26 \%$ of the total variance.

The 12-month multivariate model identified similar predictors, with longer duration (2-6 months: $22.95,13.53$ to 32.36 ; 6-12 months: $21.88,11.6$ to 32.15 ; >12 months: $24.94,15.78$ to 34.11$)$, greater baseline severity of worst or activity-related pain $(0.29,0.11$ to 0.46$)$, and lower baseline AKP Scale score $(-0.45,-0.72$ to -0.18$)$ significantly associated with greater

Table 2 Measures at 3 and 12 months $(n=310)$

\begin{tabular}{llc}
\hline Measure & 3 months & 12 months \\
\hline Pain severity (worse, activity-related) (/100) & $35.37(26.71)$ & $26.51(27.56)$ \\
Anterior Knee Pain Scale (/100) & $80.80(14.04)$ & $85.00(13.77)$ \\
Functional Index Questionnaire (/16) & $12.48(2.96)$ & $13.37(2.98)$ \\
Global recovery & & \\
$\quad$ Favourable recovery, n (\%) & $113(36.5)$ & $162(52.3)$ \\
$\quad$ Unfavourable recovery, n (\%) & $170(54.8)$ & $126(40.6)$ \\
$\quad$ Unknown, n (\%) & $27(8.7)$ & $22(7.1)$ \\
\hline Mean (SD) unless otherwise stated. & &
\end{tabular}


Table 3 Prognostic factors for pain severityt at 3 and 12 months

\begin{tabular}{|c|c|c|c|c|}
\hline \multirow[b]{2}{*}{ Variables } & \multicolumn{2}{|l|}{3 months } & \multicolumn{2}{|l|}{12 months } \\
\hline & $\boldsymbol{\beta}(95 \% \mathrm{Cl})$ & p Value & $\boldsymbol{\beta}(95 \% \mathrm{Cl})$ & p Value \\
\hline \multicolumn{5}{|l|}{ Age } \\
\hline \multicolumn{5}{|l|}{ Gender } \\
\hline \multicolumn{5}{|l|}{ Body mass index } \\
\hline \multicolumn{5}{|c|}{ Duration of complaints (ref $=1-2$ months) } \\
\hline 2-6 months & & & 22.95 (13.53 to 32.36$)$ & $<0.001$ \\
\hline $6-12$ months & 12.33 (3.56 to 21.09$)$ & 0.006 & 21.88 (11.60 to 32.15$)$ & $<0.001$ \\
\hline $12+$ months & 11.36 (3.96 to 18.77$)$ & 0.003 & 24.94 (15.78 to 34.11$)$ & $<0.001$ \\
\hline \multicolumn{5}{|c|}{ Allocated preferred treatment (ref = no preference) } \\
\hline \multicolumn{5}{|l|}{ Not allocated } \\
\hline \multicolumn{5}{|l|}{ Allocated } \\
\hline Recruitment & & & $-17.50(-25.85$ to -9.15$)$ & $<0.001$ \\
\hline \multicolumn{5}{|l|}{ Bilaterality } \\
\hline \multicolumn{5}{|l|}{ Work type (ref=not employed) } \\
\hline \multicolumn{5}{|l|}{ Sedentary } \\
\hline \multicolumn{5}{|l|}{ Active } \\
\hline \multicolumn{5}{|l|}{ Sport } \\
\hline \multicolumn{5}{|l|}{ Baseline usual/rest pain } \\
\hline Baseline worse/on activity pain & $0.45(0.28$ to 0.62$)$ & $<0.001$ & $0.29(0.11$ to 0.46$)$ & 0.001 \\
\hline Anterior Knee Pain Scale & $-0.33(-0.59$ to -0.06$)$ & 0.015 & $-0.45(-0.72$ to -0.18$)$ & 0.001 \\
\hline \multicolumn{5}{|l|}{ Functional Index Questionnaire } \\
\hline$R^{2}$ & 0.260 & & 0.237 & \\
\hline AUC (cut-off pain at follow-up 40) & 0.534 & & 0.631 & \\
\hline AUC (cut-off pain at follow-up 60) & 0.790 & & 0.736 & \\
\hline AUC (cut-off pain at follow-up 80) & 0.915 & & 0.869 & \\
\hline
\end{tabular}

12-month pain severity (table 3). Recruitment method was also included in the final model $(-17.5,-25.85$ to -9.15$)$, indicating that participants recruited by a health professional were more likely to have higher pain scores at 12 months. The multivariate model explained approximately $24 \%$ of the total variance.

\section{AKP Scale}

Table 4 presents the multivariate models for prognosis measured on the AKP Scale. The 3-month multivariate model revealed that longer PFP duration (6-12 months: $-5.35,-9.76$ to -0.94 ; > 12 months: $-4.44,-8.17$ to -0.71$)$ and lower baseline AKP Scale score $(0.56,0.44$ to 0.67$)$ were significantly associated with lower AKP Scale score at 3 months, explaining 33\% of the variance. Longer duration (2-6 months: $-8.75,-13.46$ to -4.04 ; 6-12 months: $-10.02,-15.16$ to -4.87 ; $>12$ months: $-11.33,-15.96$ to -6.71$)$ and lower baseline AKP Scale score $(0.37,0.24$ to 0.49$)$ were also significantly associated with lower 12-month AKP Scale scores. In addition, higher baseline usual or resting pain severity $(-0.1,-0.18$ to -0.02 ) was included in the 12 -month multivariate model, which explained approximately $32 \%$ of the total variance.

\section{Functional Index Questionnaire}

Multivariate analysis revealed that lower baseline AKP Scale $(0.06,0.02$ to 0.10$)$ and FIQ scores $(0.27,0.08$ to 0.47$)$ were significantly associated with lower 3-month FIQ score (table 5). This explained $27 \%$ of the total variance. The 12 -month multivariate model also included lower baseline AKP Scale score $(0.08,0.05$ to 0.11$)$, as well as greater baseline usual/resting pain severity $(-0.02,-0.04$ to -0.01$)$, longer PFP duration (2-6 months: $-1.56,-2.58$ to -0.53 ; >12 months: -1.6 , -2.46 to -0.73$)$ and female gender $(-0.71,-1.35$ to -0.07$)$. This explained $23.5 \%$ of the total variance.

\section{Global recovery}

Table 6 presents multivariate analyses for global recovery. Factors significantly associated with unfavourable recovery at 3 months were male gender (OR $0.57,95 \%$ CI 0.33 to 0.98 ), PFP duration greater than 6 months (6-12 months: $2.86,1.14$ to 7.21 ; >12 months $2.51,1.22$ to 5.16 ), no sedentary work $(0.37,0.19$ to 0.73$)$, and greater usual or resting pain severity at baseline $(1.01,1.00$ to 1.03$)$. This explained $17 \%$ of the total variance. The index of predictive discrimination for this model (area under the curve) was 0.71 , reflecting moderate ability of the model to discriminate between patients with favourable and unfavourable recovery. Longer duration (2-6 months: 4.04, 1.66 to 9.82 ; $6-12$ months: $4.10,1.62$ to $10.40 ;>12$ months: $6.11,2.56$ to 14.59$)$ and recruitment by a health professional $(0.50,0.28$ to 0.92$)$ were significantly associated with unfavourable 12 -month recovery, explaining $9 \%$ of the total variance. The accuracy of the model was moderate with an area under the curve of 0.65 .

\section{Baseline predictor values associated with unfavourable recovery at 12 months}

From the multivariate models, baseline PFP duration greater than 2 months was associated with unfavourable recovery at 12 months. Baseline AKP Scale score of less than 70/100, as well as pain severity of greater than $35 / 100$ (usual/resting) and 
Table 4 Prognostic factors for Anterior Knee Pain Scale at 3 and 12 months

\begin{tabular}{|c|c|c|c|c|}
\hline \multirow[b]{2}{*}{ Variables } & \multicolumn{2}{|l|}{3 months } & \multicolumn{2}{|l|}{12 months } \\
\hline & $\boldsymbol{\beta}(95 \% \mathrm{Cl})$ & $\mathrm{p}$ Value & B $(95 \% \mathrm{Cl})$ & p Value \\
\hline \multicolumn{5}{|l|}{ Age } \\
\hline \multicolumn{5}{|l|}{ Gender } \\
\hline \multicolumn{5}{|l|}{ Body mass index } \\
\hline \multicolumn{5}{|c|}{ Duration of complaints (ref $=1-2$ months) } \\
\hline 2-6 months & & & $-8.75(-13.46$ to -4.04$)$ & $<0.001$ \\
\hline 6-12 months & $-5.35(-9.76$ to -0.94$)$ & 0.021 & $-10.02(-15.16$ to -4.87$)$ & $<0.001$ \\
\hline $12+$ months & $-4.44(-8.17$ to -0.71$)$ & 0.024 & $-11.33(-15.96$ to -6.71$)$ & $<0.001$ \\
\hline \multicolumn{5}{|c|}{ Allocated preferred treatment (ref $=$ no preference) } \\
\hline \multicolumn{5}{|c|}{ Not allocated } \\
\hline \multicolumn{5}{|l|}{ Allocated } \\
\hline \multicolumn{5}{|l|}{ Recruitment } \\
\hline \multicolumn{5}{|l|}{ Bilaterality } \\
\hline \multicolumn{5}{|l|}{ Work type (ref=not employed) } \\
\hline \multicolumn{5}{|l|}{ Sedentary } \\
\hline \multicolumn{5}{|l|}{ Active } \\
\hline \multicolumn{5}{|l|}{ Sport } \\
\hline Baseline usual/rest pain & & & $-0.099(-0.18$ to -0.02$)$ & 0.015 \\
\hline \multicolumn{5}{|l|}{ Baseline worse/on activity pain } \\
\hline Anterior Knee Pain (AKP) Scale & $0.56(0.44$ to 0.67$)$ & $<0.001$ & $0.37(0.24$ to 0.49$)$ & $<0.001$ \\
\hline \multicolumn{5}{|l|}{ Functional Index Questionnaire } \\
\hline $\mathrm{R}^{2}$ & 0.330 & & 0.317 & \\
\hline AUC (cut-off AKP at follow-up 90) & 0.550 & & 0.656 & \\
\hline AUC (cut-off AKP at follow-up 80) & 0.675 & & 0.304 & \\
\hline AUC (cut-off AKP at follow-up 60) & 0.101 & & 0.085 & \\
\hline
\end{tabular}

Variable 'treatment' not presented, but was included in final models (significant in 3-month and 12-month model), Backward selection: $p(\mathrm{IN})=0.05, p(\mathrm{OUT})=0.10$.

Table 5 Prognostic factors for Functional Index Questionnaire score at 3 and 12 months

\begin{tabular}{|c|c|c|c|c|}
\hline \multirow[b]{2}{*}{ Variables } & \multicolumn{2}{|l|}{3 months } & \multicolumn{2}{|l|}{12 months } \\
\hline & $\boldsymbol{\beta}(95 \% \mathrm{Cl})$ & $\mathrm{p}$ Value & $\boldsymbol{\beta}(95 \% \mathrm{Cl})$ & p Value \\
\hline \multicolumn{5}{|l|}{ Age } \\
\hline Gender & & & $-0.71(-1.35$ to -0.07$)$ & 0.03 \\
\hline \multicolumn{5}{|l|}{ Body mass index } \\
\hline \multicolumn{5}{|c|}{ Duration of complaints (ref=1-2 months) } \\
\hline 2-6 months & & & $-1.56(-2.58$ to -0.53$)$ & 0.03 \\
\hline $6-12$ months & & & $-1.03(-2.09$ to 0.03$)$ & 0.056 \\
\hline $12+$ months & & & $-1.60(-2.46$ to -0.73$)$ & $<0.001$ \\
\hline \multicolumn{5}{|c|}{ Allocated preferred treatment (ref=no preference) } \\
\hline \multicolumn{5}{|l|}{ Not allocated } \\
\hline \multicolumn{5}{|l|}{ Allocated } \\
\hline \multicolumn{5}{|l|}{ Recruitment } \\
\hline \multicolumn{5}{|l|}{ Bilaterality } \\
\hline \multicolumn{5}{|l|}{ Work type (ref = not employed) } \\
\hline \multicolumn{5}{|l|}{ Sedentary } \\
\hline \multicolumn{5}{|l|}{ Active } \\
\hline \multicolumn{5}{|l|}{ Sport } \\
\hline Baseline usual/rest pain & $-0.02(-0.03$ to 0.002$)$ & 0.08 & $-0.02(-0.04$ to -0.01$)$ & 0.013 \\
\hline \multicolumn{5}{|l|}{ Baseline worse/on activity pain } \\
\hline Anterior Knee Pain Scale & $0.06(0.02$ to 0.10$)$ & 0.001 & $0.08(0.05$ to 0.11$)$ & $<0.001$ \\
\hline Functional Index Questionnaire & 0.27 (0.08 to 0.47$)$ & 0.007 & & \\
\hline$R^{2}$ & 0.273 & & 0.235 & \\
\hline AUC (cut-off FIQ at follow-up 12) & 0.509 & & 0.405 & \\
\hline AUC (cut-off FIQ at follow-up 14) & 0.611 & & 0.563 & \\
\hline
\end{tabular}

Variable 'treatment' not presented, but was included in final models (significant in 3-month and 12-month model).

Backward selection: $p(\mathrm{IN})=0.05, \mathrm{p}(\mathrm{OUT})=0.10$. 
Table 6 Prognostic factors for unfavourable recovery at 3 and 12 months

\begin{tabular}{|c|c|c|c|c|}
\hline \multirow[b]{2}{*}{ Variables } & \multicolumn{2}{|l|}{3 months } & \multicolumn{2}{|l|}{12 months } \\
\hline & OR $(95 \% \mathrm{Cl})$ & p Value & OR $(95 \% \mathrm{Cl})$ & p Value \\
\hline \multicolumn{5}{|l|}{ Age } \\
\hline Gender & $0.57(0.33$ to 0.98$)$ & 0.040 & & \\
\hline \multicolumn{5}{|c|}{ Body mass index } \\
\hline \multicolumn{5}{|c|}{ Duration of complaints (ref $=1-2$ months) } \\
\hline 2-6 months & 2.32 (0.99 to 5.38$)$ & 0.051 & $4.04(1.66$ to 9.82$)$ & 0.002 \\
\hline 6-12 months & 2.86 (1.14 to 7.21$)$ & 0.026 & 4.10 (1.62 to 10.40$)$ & 0.003 \\
\hline $12+$ months & 2.51 (1.22 to 5.16$)$ & 0.013 & $6.11(2.56$ to 14.59$)$ & $<0.001$ \\
\hline \multicolumn{5}{|c|}{ Allocated preferred treatment (ref = no preference) } \\
\hline \multicolumn{5}{|l|}{ Not allocated } \\
\hline \multicolumn{5}{|l|}{ Allocated } \\
\hline Recruitment & & & $0.50(0.28$ to 0.92$)$ & 0.025 \\
\hline Bilaterality & 1.69 (0.99 to 2.87$)$ & 0.054 & & \\
\hline \multicolumn{5}{|c|}{ Work type (ref=not employed) } \\
\hline Sedentary & $0.37(0.19$ to 0.73$)$ & 0.004 & & \\
\hline Active & $0.51(0.26$ to 1.03$)$ & 0.06 & & \\
\hline \multicolumn{5}{|l|}{ Sport } \\
\hline Baseline usual/rest pain & 1.01 (1.00 to 1.03$)$ & 0.043 & & \\
\hline \multicolumn{5}{|c|}{ Baseline worse/on activity pain } \\
\hline \multicolumn{5}{|l|}{ Anterior Knee Pain Scale } \\
\hline \multicolumn{5}{|c|}{ Functional Index Questionnaire } \\
\hline $\mathrm{R}^{2}$ & 0.17 & & 0.09 & \\
\hline AUC & 0.706 & & 0.648 & \\
\hline
\end{tabular}

greater than $60 / 100$ (worst/activity-related), corresponded to unfavourable recovery at 12 months.

\section{DISCUSSION}

An important finding of this study was that, of 310 participants with PFP, 40\% reported unfavourable 12-month recovery (rated as global recovery of 'moderate improvement' to 'worse than ever'). This was higher than the proportion randomly allocated to either of the RCT minimal intervention or control arms (110/310, 35\%), and highlights that PFP is not self-limiting, even in a mixed cohort who have undergone effective interventions. $^{2} 5$ Interestingly, the majority of participants who experienced a favourable recovery had already reached this within the first 3 months. This confirms an earlier study that reported greatest improvement in PFP and function during the initial 3 months. $^{26}$ Furthermore, Witvrouw et al ${ }^{26}$ reported a 5-year AKP Scale score similar to our findings at 12 months. Taken together, these findings suggest that the improvements in knee symptoms observed following 5-6 weeks intervention, may plateau beyond this time. Considering that persistent pain can negatively affect daily and occupational tasks, physical activity, social participation and general and mental health, the implications of chronic PFP require further investigation.

Across the four outcome measures investigated, the most consistent short-term and long-term prognostic factors were longer PFP duration and lower AKP Scale score. Specifically, those who reported PFP of greater than 2 months duration and AKP Scale score less than $70 / 100$ had a poorer 12 -month prognosis. Pain severity measured on a severity measured on a VAS was also identified as a frequent prognostic factor, being usual or resting pain severity greater than $35 / 100$, and worst or activity-related pain severity greater than $60 / 100$. These findings concur with our previous analysis involving a smaller proportion of this PFP cohort,$~^{10}$ and with other musculoskeletal conditions. ${ }^{9}$

There were additional variables identified as prognostic factors on particular measures at particular times. While these are interesting and hypothesis-generating, they need to be investigated further before being considered as prognostic factors. For example, participants recruited via a health professional tended to have a poorer prognosis. This may be related to baseline symptom severity, in that those with worse symptoms may be more likely to visit a health professional. Interestingly, conflicting results were found for the prognostic factor gender for the different time periods and on different measures. Males tended to have a poorer prognosis at 3 months on the measure of global recovery, while females had poorer function at 12 months as measured on the FIQ, perhaps reflecting gender differences in temporal pain patterns. Finally, those with a sedentary occupation were less likely to report an unfavourable outcome compared to non-employees at 3 months, possibly due to differences in daily knee loading patterns. While these factors should be considered when evaluating individual patient prognosis, less consistent findings regarding their prognostic ability means that they should be regarded as secondary predictors until further studies have been conducted.

It is important to consider that the predictors investigated accounted for a smaller percentage of total variance for the global recovery score than for outcomes of pain and/or function. It is known that in patients with acute lateral ankle sprains, favourable recovery can be partly explained by other symptoms (eg, instability). ${ }^{27}$ This indicates that other variables interact with the more general measure of global recovery. A consideration in this PFP cohort is that other variables not investigated may have a greater influence on perceived global recovery than 
on measures of pain and function. These may include muscle strength, proprioception, kinesiophobia, quality of life, psychological health and pain characteristics such as central sensitisation, and warrant consideration in design of future prognostic studies. Furthermore, classifications of 'favourable' and 'unfavourable' recovery were made by the investigators based on participant responses, rather than by the participants themselves. More insight is needed into the meaning of a 'favourable recovery' in PFP, and to reach consensus about implications for clinical practice.

Interestingly, more than two-thirds of participants in the Australian RCT, who were recruited primarily via self-referral, reported PFP duration of 12 months or longer. Additionally, at least $25 \%$ of those recruited by health professionals in the Netherlands had their knee pain for 6 months or longer before they visited their health professional. On the basis of this long symptom duration in many of the Dutch and Australian participants, it is apparent that the general public and health professionals (eg, general and sports physicians, orthopaedists, physiotherapists) require education regarding the importance of early intervention to maximise prognosis for those with PFP. Furthermore, utilisation of interventions with known efficacy to reduce symptoms, ${ }^{28}$ early in the course of the condition, may enhance PFP prognosis. Considering the proposed relationship between PFP and PFJ osteoarthritis in later life, ${ }^{4}$ this may have important implications for minimising the impact of chronic musculoskeletal disease.

\section{Strengths and limitations}

This study utilised data from 310 participants in two highquality RCTs, and represents the largest cohort of PFP participants. Additionally, this study has attempted to address methodological limitations of previous prognostic studies, and is the first to quantify baseline scores that may highlight poor prognosis. Despite differences between the two cohorts (eg, recruitment method), this study identified from the pooled dataset prognostic factors that are able to consistently identify those with a poorer prognosis over 12 months.

While this study represents an important step in identifying PFP prognostic factors, there are limitations to acknowledge. First, this post hoc analysis of RCTs was not the optimal design to evaluate prognosis, and the sample size not powered for prognostic analysis. Nevertheless, we addressed this limitation by restricting candidate variables to a maximum of 13 for multivariate modeling. This adhered to 'the rule of ten' (type I error), meaning that we did not enter more than one variable per 10 PFP participants with a favourable recovery. A further consequence of the study design was that six different interventions were provided to participants across the two RCTs. We attempted to adjust for differing treatment effects on follow-up measures by entering treatment as a factor in all analyses. Finally, because the RCTs were not designed to investigate prognostic factors, potential predictor variables investigated were limited to those measured consistently by both studies at baseline. As such, we were unable to investigate other variables that may have influenced prognosis, such as health-related quality of life, physical activity, muscle strength and kinesiophobia.

\section{CONCLUSIONS}

A substantial proportion of individuals with PFP have an unfavourable recovery over 12 months, irrespective of intervention. Duration of PFP greater than 2 months is the most consistent predictor of poor long-term prognosis, along with a score less than 70 on the AKP Scale. Those that report higher levels of usual/resting or worst/activity-related pain should also be flagged as potentially having a poor 12 -month prognosis. Sports medicine practitioners should promote education regarding the natural history and importance of early intervention for PFP, and prescribe interventions with known efficacy in reducing PFP, in order to maximise prognosis.

\section{What are the new findings}

- A substantial proportion of people with patellofemoral pain (PFP) experience an unfavourable outcome over 12 months, irrespective of intervention.

- PFP of greater than 2 months duration at baseline is the most consistent predictor of poor outcome.

- An Anterior Knee Pain Scale score less than 70/100, indicating worse pain and function, is also a consistent prognostic factor.

- Findings suggest that early management utilising interventions with known efficacy in reducing PFP may enhance prognosis.

Contributors NC, SBZ, KC, BV and MVM designed the study. NC, MVM and RVL collected data for the original studies. NC and MVM performed statistical analyses. NC, SBZ, KC and MVM drafted the manuscript. All authors reviewed and approved the final manuscript.

Funding The Australian trial was funded by the National Health and Medical Research Council (NHMRC) of Australia (Primary Health Care project grant No 301037). The Dutch trial was funded by The Netherlands Organisation for Health Research and Development (ZON-MW). Dutch trial grant number (ZON-MW) is 945-04-356. NC is supported by a NHMRC Health Professional Research Training (Post-Doctoral) Fellowship (No 628918).

Competing interests None.

Ethics approval The University of Queensland's Medical Research Ethics Committee; The Erasmus Medical University, Rotterdam.

Provenance and peer review Not commissioned; externally peer reviewed.

\section{REFERENCES}

1 Crossley K, Bennell K, Green S, et al. Physical therapy for patellofemoral pain: a randomized, double-blinded, placebo-controlled trial. Am J Sports Med 2002;30:857-65

2 Collins N, Crossley K, Beller E, et al. Foot orthoses and physiotherapy in the treatment of patellofemoral pain syndrome: randomized clinical trial. $B M J$ 2008;337:a1735.

3 Nimon G, Murray D, Sandow M, et al. Natural history of anterior knee pain: a 14- to 20-year follow-up of nonoperative management. J Pediatr Orthop 1998; 18:118-22.

4 Thomas MJ, Wood L, Selfe J, et al. Anterior knee pain in younger adults as a precursor to subsequent patellofemoral osteoarthritis: a systematic review. BMC Musculoskelet Disord 2010;11:201.

5 van Linschoten R, van Middelkoop M, Berger MY, et al. Supervised exercise therapy versus usual care for patellofemoral pain syndrome: an open label randomized controlled trial. BMJ 2009;339:b4074.

6 Jewell DV. Guide to evidence-based physical therapy practice. Sudbury, MA: Jones and Bartlett Publishers, Inc, 2008.

7 Moons KG, Royston P, Vergouwe $Y$, et al. Prognosis and prognostic research: what, why, and how? BMJ 2009;338:b375.

8 Straus SE, Richardson WS, Glasziou P, et al. Evidence-based medicine. How to practice and teach EBM. 3rd edn. Edinburgh: Elsevier, 2005.

9 Mallen $C D$, Peat $G$, Thomas $E$, et al. Prognostic factors for musculoskeletal pain in primary care: a systematic review. Br J Gen Pract 2007;57:655-61.

10 Collins NJ, Crossley KM, Darnell R, et al. Predictors of short and long term outcome in patellofemoral pain syndrome: a prospective longitudinal study. BMC Musculoskelet Disord 2010;11:11.

11 Kannus P, Niittymaki S. Which factors predict outcome in the nonoperative treatment of patellofemoral pain syndrome? A prospective follow-up study. Med Sci Sports Exerc 1994;26:289-96. 
12 Natri A, Kannus $\mathrm{P}$, Jarvinen $\mathrm{M}$. Which factors predict the long-term outcome in chronic patellofemoral pain syndrome? A 7-yr prospective follow-up study. Med Sci Sports Exerc 1998;30:1572-7.

13 Witvrouw E, Lysens R, Bellemans J, et al. Which factors predict outcome in the treatment program of anterior knee pain? Scand J Med Sci Sports 2002:12:40-6.

14 van Linschoten $\mathrm{R}$, van Middelkoop M, Berger MY, et al. The PEX study-exercise therapy for patellofemoral pain syndrome: design of a randomized clinical trial in general practice and sports medicine. BMC Musculoskelet Disord 2006;7:31.

15 Vicenzino B, Collins N, Crossley K, et al. Foot orthoses and physiotherapy in the treatment of patellofemoral pain syndrome: a randomized clinical trial. $B M C$ Musculoskelet Disord 2008;9:27.

16 Kujala UM, Jaakkola LH, Koskinen SK, et al. Scoring of patellofemoral disorders. Arthroscopy 1993;9:159-63.

17 Chesworth BM, Culham EG, Tata GE, et al. Validation of outcome measures in patients with patellofemoral syndrome. J Orthop Sports Phys Ther 1989;10:302-8.

18 Hoving JL, Koes BW, de Vet HC, et al. Manual therapy, physical therapy, or continued care by a general practitioner for patients with neck pain. A randomized, controlled trial. Ann Intern Med 2002;136:713-22.

19 Vonk F, Verhagen AP, Geilen M, et al. Effectiveness of behavioural graded activity compared with physiotherapy treatment in chronic neck pain: design of a randomized clinical trial (ISRCTN88733332). BMC Musculoskelet Disord 2004:5:34.
20 Peduzzi $\mathrm{P}$, Concato J, Kemper $\mathrm{E}$, et al. A simulation study of the number of events per variable in logistic regression analysis. J Clin Epidemiol 1996;49:1373-9.

21 Royston P, Altman DG, Sauerbrei W. Dichotomizing continuous predictors in multiple regression: a bad idea. Stat Med 2006;25:127-41.

22 Royston $\mathrm{P}$, Moons KG, Altman DG, et al. Prognosis and prognostic research: developing a prognostic model. BMJ 2009;338:b604.

23 Harrell FE Jr, Lee $\mathrm{KL}$, Mark DB. Multivariable prognostic models: issues in developing models, evaluating assumptions and adequacy, and measuring and reducing errors. Stat Med 1996;15:361-87.

24 Zweig MH, Campbell G. Receiver-operating characteristic (ROC) plots: a fundamental evaluation tool in clinical medicine. Clin Chem 1993;39:561-77.

25 Koch HJ, Hau P. ROC analysis as an additional method to characterize time to event data. Pathol Oncol Res 2005;11:50-2.

26 Witvrouw E, Danneels L, Van Tiggelen D, et al. Open versus closed kinetic chain exercises in patellofemoral pain: a 5-year prospective randomized study. Am J Sports Med 2004;32:1122-30.

27 van Rijn RM, van Os AG, Bernsen RM, et al. What is the clinical course of acute ankle sprains? A systematic literature review. Am J Med 2008;121:324e6-31e6.

28 Collins NJ, Bisset LM, Crossley KM, et al. Efficacy of non-surgical interventions for anterior knee pain: systematic review and meta-analysis of randomized trials. Sports Med 2012:42:31-49. 